\title{
SEMIGROUP GENERATIONS OF UNBOUNDED BLOCK OPERATOR MATRICES BASED ON THE SPACE DECOMPOSITION
}

\author{
Jie LiU, Junjie Huang And Alatancang Chen
}

Abstract. This paper deals with the problem for unbounded block operator matrix

$$
M=\left(\begin{array}{ll}
A & B \\
C & D
\end{array}\right)
$$

with natural domain to generate $C_{0}$ semigroups, based on the space decomposition. By describing the spectral inclusion relations between the numerical range of $M$ and its inner entries, using the quadratic complements of $M$, some necessary and sufficient conditions for $M$ to generate $C_{0}$ semigroups are given.

Mathematics subject classification (2010): 47D03, 47B99, 37K99. sition.

Keywords and phrases: Unbounded block operator matrix, generator, $C_{0}$ semigroup, space decompo-

\section{REFERENCES}

[1] Goldstein J. A., Semigroups of Linear Operators and Applications, Oxford University Press, New York, 1985.

[2] Engel K. J., NAGEl R., One Parameter Semigroups for Linear Evolution Equations, Graduate Texts in Mathematics 194, Springer-Verlag, New York, 2000.

[3] NAGEL R., Towards a "matrix theory" for unbounded operator matrices, Math. Z, 1989, 201, 57-68.

[4] Mugnolo D., Asymptotics of semigroups generated by operator matrices, Arab. J. Math. 2014, 3: $419-435$.

[5] Kato T., Perturbation Theory for Linear Operators, Classics in Mathematics, Springer-Verlag, Berlin, 1995.

[6] Langer H., Markus A. S., Matsaev V. I., Tretter C., A new concept for block operator matrices: the quadratic numerical range, Linear Algebra Appl., 330 (1-3), 2001, 89-112.

[7] Tretter C., Spectral Theory of Block Operator Matrices and Applications, Imperial College Press, London, 2008.

[8] Tretter C., Spectral inclusion for unbounded block operator matrices, Journal of Functional Analysis 256, 2009, 3806-3829.

[9] Wu D., Chen A., Spectral inclusion properties of the numerical range in a space with an indefinite metric, Linear Algebra Appl, 2011, 435: 1131-1136.

[10] Krein S. G., Linear Differential Equations in Banach Space, American Mathematical Society, Providence, Rhode Island, 1971. 